\title{
Evoluçáo natural em uma paciente com Mucopolissacaridose tipo VI
}

\author{
Natural evolution in a patient with Mucopolysaccharidosis type VI \\ Juliana Olavo Pereira ${ }^{1}$, Maria Gabriela de Aguiar Moreira \\ Garbelini ${ }^{1}$, Vitor Costa Palazzo ${ }^{2}$
}

\begin{abstract}
RESUMO
Introduçáo. A mucopolissacaridose tipo VI é causada pela deficiência da n-acetilgalactosamina-4-sulfatase, uma das onze enzimas lisossomais responsáveis pela degradaçáo de glicosaminoglicanos, e estas, ao se acumularem causam dano ao lisossomo, morte celular e disfunção orgânica. Objetivo. Apresentar a evolução natural da deposição dos glicosaminoglicanos em uma paciente sem tratamento prévio e relacionar as dificuldades na conduta e diagnóstico desta rara doença no Brasil. Relato do Caso. Paciente apresentando quadro multissistêmico devido à mucopolissacaridose tipo VI após quatorze anos da doença, sem tratamento ou acompanhamento. Conclusáo. $\mathrm{O}$ diagnóstico precoce e a terapêutica correta são juntos indispensáveis para uma qualidade de vida a esses pacientes.
\end{abstract}

Unitermos. Mucopolissacaridose VI, Síndrome de Maroteaux-Lamy, Doenças por Armazenamento dos Lisossomos, Glicosaminoglicanos, Erros Inatos do Metabolismo.

Citaçáo. Pereira JO, Garbelini MGAM, Palazzo VC. Evolução natural em uma paciente com Mucopolissacaridose tipo VI.

\begin{abstract}
Introduction. The mucopolysaccharidosis type VI is caused by deficiency of $n$-acetilgalactosamina-4-sulfatase, one of eleven lysosomal enzymes responsible for degradation of glycosaminoglycans, and these, when combined cause damage to the lysosomes, cell death and organic dysfunction. Objective. To present the natural evolution of the deposition of glycosaminoglycans in a patient without prior treatment and relate the difficulties in the conduct and diagnosis of this rare disease in Brazil. Case Report. Patient presenting multisystemic framework due to mucopolysaccharidosis type VI after fourteen years of disease, without treatment or monitoring. Conclusion. Early diagnosis and correct therapy are together essential for a quality of life for these patients.
\end{abstract}

Keywords. Mucopolysaccharidosis VI; Maroteaux-Lamy Syndrome; Lysosomal Storage Diseases; Glycosaminoglycans; Metabolism Inborn Errors.

Citation. Pereira JO, Garbelini MGAM, Palazzo VC. Natural evolution in a patient with Mucopolysaccharidosis type VI.
Endereço para correspondência: Juliana O Pereira R Percy Withers, 121, apto 72. CEP 80240-190, Curitiba-PR, Brasil.

Tel.: 3244-3755.

E-mail: julianaolavopereira@gmail.com
Trabalho realizado no Hospital Pequeno Príncipe - Curitiba-PR, Brasil. 1. Discente do curso de medicina, da Universidade Positivo, Curitiba-PR, Brasil. 2. Pediatra, Docente da disciplina de Pediatria e Puericultura, da Universidade Positivo, Curitiba-PR, Brasil.
Relato de Caso Recebido em: 03/09/09 Aceito em: 02/06/10 Conflito de interesses: não 


\section{INTRODUÇÃO}

A Mucopolissacaridose VI, também conhecida como síndrome de Maroteaux-Lamy, é uma doença metabólica autossômica recessiva rara, devido à deficiência da enzima n-acetilgalactosamina-4-sulfatase ou arilsulfatase B (ARSB), o que leva o acúmulo dos glicosaminoglicanos (GAG's) sulfato de dermatina e sulfato de condroitina em órgãos e tecidos. Está incluída entre os denominados erros inatos do metabolismo (EIM), fazendo parte das doenças de depósito lisossomal ${ }^{1-6}$.

Apresenta incidência mundial variável (1:238.095 a $1: 300.000)^{4}$. Estudos em alguns países não apresentam nenhum caso em mais de vinte anos, mas estudos brasileiros encontraram incidências altas, levando-se a considerar que sua incidência seja maior no Brasil ${ }^{1}$. A MPS Brazil Network, uma iniciativa para melhorar o diagnóstico e manejo das mucopolissacaridoses (MPS), identificou 249 pacientes entre abril de 2004 e setembro de 2006, sendo 57 com MPS VI, 80 com MPS II e 60 com MPS I7.

A suspeita diagnóstica baseia-se na clínica compatível e a investigação inicial é através da constatação de mucopolissacaridúria no teste azul de toluidina e na cromatografia. A confirmação é dada pela redução da atividade da enzima $(<10 \%)$ em leucócitos ou fibroblastos ou pela mutação genética no gene ARSB do cromossomo $5 \mathrm{q} 13-14^{1,4,7,8}$.

O quadro clínico é multissistêmico e bastante variável. O desenvolvimento cognitivo pode estar preservado, assim como o pôndero-estatural, mas frequentemente ocorre baixa estatura. $\mathrm{O}$ acúmulo de glicosaminoglicanos no conduto auditivo pode levar a otites crônicas, com conseqüente deformidades dos ossículos e hipoacusia. $\mathrm{O}$ excesso de GAGs nos olhos pode causar opacificação de córnea, glaucoma, "pseudoglaucoma” e papiledema, sendo que em crianças pode apresentar diminuição importante da acuidade visual e amaurose total nos casos mais graves. O sistema cardiovascular pode ser afetado através de patologias valvulares, insuficiência cardíaca congestiva, hipertensão arterial sistêmica e hipertensão pulmonar, sendo esta última a complicação de maior morbidade. São comuns infecções respiratórias de repetição até apnéia obstrutiva do sono, pelo acometimento do sistema respiratório. Como o acúmulo de GAGs pode ocorrer em vários órgãos e tecidos, o paciente pode apresentar hepa- toesplenomegalia, infiltração de extremidades, alteraçóes cutâneas, displasia dentária, macroglossia, hipertricose, fácies grosseira típica. Além disso, pode ocorrer hidrocefalia, atrofia cerebral, ventriculomegalia, alterações no sistema nervoso autonômico e mielopatia por compressão. São extremamente comuns alteraçóes esqueléticas, conhecidas como disostose múltipla, sendo que o paciente apresenta função articular limitada, mão em garra, síndrome do túnel do carpo ${ }^{1,2,4,5,8-12}$.

O tratamento de escolha é a terapia de reposição enzimática (TRE) com galsulfase $1 \mathrm{mg} / \mathrm{kg}$, administrada uma vez por semana, com infusão intravenosa de duração de quatro horas. Os ensaios clínicos e estudos a longo prazo demonstraram importante redução das GAGs urinárias, com melhora significativa da resistência física dos pacientes e diminuição da progressão da doença. A segurança foi comprovada com uma pequena taxa de reaçóes adversas graves ${ }^{1,3,4,13-18}$.

Os objetivos deste trabalho são apresentar a evolução natural da deposição dos GAG’s em uma paciente sem tratamento prévio.

\section{RELATO DE CASO}

V.A.H., 14 anos, diagnosticada como portadora de mucopolissacaridose há três anos, durante investigação de baixa estatura, estava sem acompanhamento ou tratamento específico. Foi internada no Hospital Pequeno Príncipe para investigação adequada e realização de exames para protocolo de reposição enzimática.

O primeiro sinal da doença foi deformidade da coluna vertebral, com um ano e meio de idade. Aos quatro, notou-se déficit auditivo. Apresentou infecções de vias aéreas de repetição, como pneumonias, otites e amigdalites, e foi submetida a uma série de cirurgias, por síndrome do túnel do carpo, displasia de quadril e hérnia umbilical. Também houve atraso do desenvolvimento neuropsicomotor, engatinhou e realizou controle esfincteriano com dois anos e andou e falou com quatro anos.

Ao exame inicial, apresentava macrocefalia (PC: $52,5)$, déficit pondero-estatural $(1,09 \mathrm{~cm}$ e $19 \mathrm{~kg})$, pele xerófita, fácies e cabelos grosseiros, hipertelorismo, macrocefalia, hipertrofia gengival e de amígdalas, má oclusão dentária, protrusão dos incisivos e respiração bucal (Figura 1). Não apresentava alteraçôes na marcha, fala ou linguagem. 
Além disso, havia hepatoesplenomegaliae hérnia umbilical. A ausculta cardíaca evidenciou sopro sistólico ++/IV. Os membros mostravam deformidades compatíveis com disostose múltipla, rigidez articular, mão em garra, unhas em vidro de relógio, além de escoliose e cifose. Força, tônus, movimentos e coordenação estavam preservados, porém com hiperreflexia global. $\mathrm{O}$ exame neurológico demonstrou presença de alteraçáo no oitavo par craniano, pela diminuição da acuidade auditiva e no segundo par devido à opacidade de córnea bilateralmente $++/ \mathrm{IV}$, além de aumento da pressão ocular $(26 \mathrm{mmHg}$ em AO).

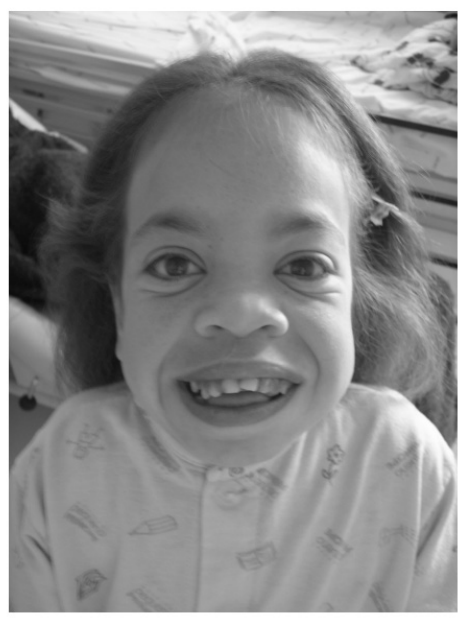

Figura 1. Fácies e cabelos grosseiros, hipertelorismo, macrocefalia, opacidade corneana, hipertrofia gengival, mal-formação dentária e macroglossia. Imagem autorizada pela responsável legal da paciente.

A ressonância magnética do encéfalo demonstrou alterações compatíveis com a doença de base (Figura 2).

As radiografias confirmaram as alteraçôes encontradas no exame físico, demonstrando a presença de escoliose tóraco-lombar, sinais sugestivos de subluxação do quadril, hipoplasia de cabeças e colos femorais, alargamento generalizado das diáfises, deformidade da regiáo meta-epifisária dos úmeros, rádio e ulnas. A radiografia de cavum demonstrou moderado aumento de partes moles, com coluna aérea reduzida, porém permeável. A idade óssea estimada foi em torno dos dez anos, porém deformidades das falanges e metacarpos prejudicaram a avaliação.

As seguintes alterações cardíacas foram demonstradas no ecocardiograma: insuficiência mitral moderada, estenose mitral leve, músculos papilares fusionados, vál- vula aórtica bicúspide, insuficiência aórtica leve, insuficiência pulmonar leve, ventrículo esquerdo globoso com paredes pouco infiltradas, porém com função mantida.

Nos testes que detectam a presença de GAGs na urina, o teste do azul de toluidina foi positivo, a cromatografia demonstrou quantidades elevadas de sulfato de dermatina e a dosagem de glicosaminoglicanos foi de $609 \mu$ GAGs/mg creatinina (VR: 26-97).

Os testes enzimáticos no sangue evidenciaram baixa atividade da arilsulfatase B em leucócitos no valor de 9,0 nmoles/h/mg proteína (VR: 72-176), confirmando a doença.

O tratamento proposto foi a terapia por reposição enzimática com galsulfase, instituída ambulatorialmente. $\mathrm{O}$ aumento da pressão ocular foi tratado com maleato de timolol a criança foi encaminhada para cirurgia pediátrica para realização de herniorrafia umbilical, adenoamigdalectomia e colocação de tubo de ventilação.

$\mathrm{O}$ relato de caso foi aprovado no comitê de ética em pesquisa do Hospital Pequeno Príncipe em Curitiba sob o número de registro 0940-11.

\section{DISCUSSÃO}

A história natural da mucopolissacadose tipo VI não é diferente do relatado nesta paciente t,2,4,5,8-12 $^{\text {. }}$

Das alteraçóes mais comuns encontradas em um estudo que avaliou nove pacientes com MPS VI ${ }^{11}$, esta apresenta quase todas as alteraçóes citadas: baixa estatura, opacidade corneana, hepatoesplenomegalia, giba tóraco-lombar, cardiopatia, rigidez articular, hirsutismo, disostose múltipla e fácies grosseira, apenas não manifesta um quadro de retardo metal franco. A paciente tem um desenvolvimento cognitivo próximo à normalidade, mas suas limitaçôes físicas e falta de estímulo adequado a impossibilitaram de se adequar à sua idade cronológica.

Em um estudo sobre as condiçóes diagnósticas da MPS no Brasil, verificou que a faixa de idade de aparecimento dos sintomas dos pacientes com MPS tipo VI foi de 0 a 24 meses $^{7}$, coincidindo com o caso da paciente, que teve seus primeiros sintomas aos 18 meses. A primeira manifestação foi óssea, como $60 \%$ dos pacientes estudados ${ }^{7}$. As principais cirurgias realizadas foram herniorrafia umbilical, adenoidectomia, tonsiloidectomia, colocação de tubo auditivo, cirurgia ortopédica e hernior- 


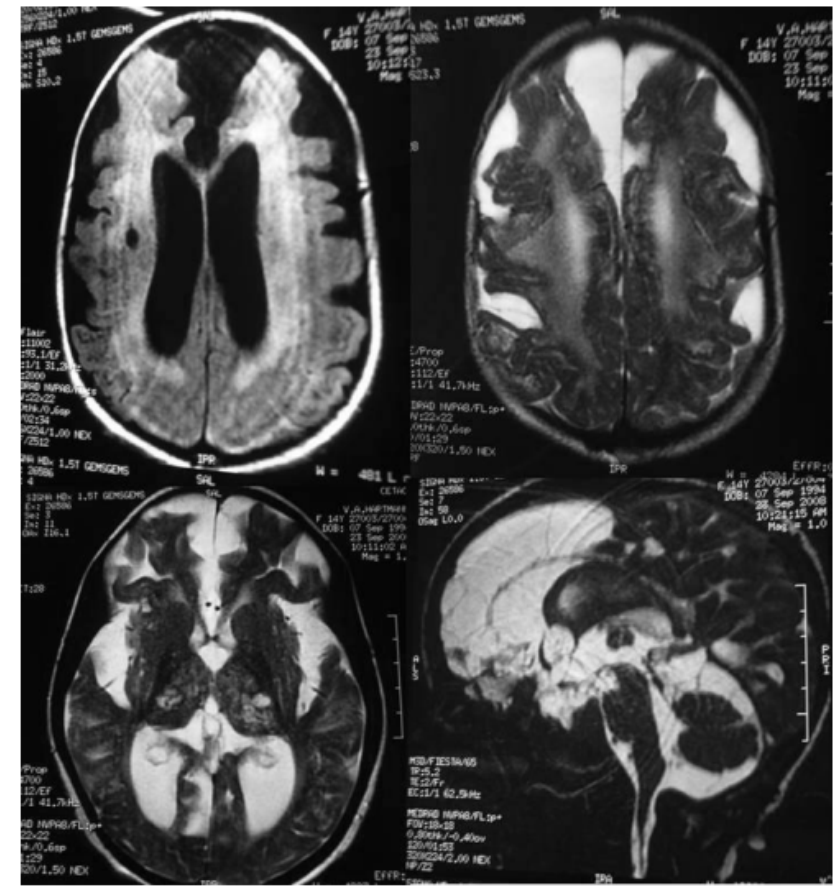

Figura 2. RNM de encéfalo: alargamento do espaço aracnóide associado à septaçóes aracnóides (cisto aracnóides), expandindo alguns sulcos corticais nas convexidades fronto-temporo-parietais bilateralmente. Extensas alterações de sinais na substância branca supratentorial, com caracteristicas inespecíficas, possivelmente indicando áreas de gliose ou desmielinização. Sela túrcica alargada e com deformação em forma de "J" e estenose moderada do forâmen magno. Difusa dilatação de espaços periventriculares e região supratentorial com aspecto multicístico nas regiốes gânglio-capsulares e nos tálamos bilateralmente.

rafia inguinal ${ }^{7}$, sendo que a paciente relatada se submeteu a todas as cirurgias citadas, exceto a herniorrafia inguinal. A paciente também apresentou atraso no desenvolvimento neuropsicomotor, como foi constatado em 2,61\%7.

As alteraçôes dos exames complementares da paciente também são características da doença, como demonstrado na literatura, revelando o quadro de disostose múltipla, cardiopatia e oftalmopatia ${ }^{1,4,8}$.

$\mathrm{O}$ estudo que avaliou as condiçóes diagnósticas dos pacientes com MPS no Brasil, conclui que normalmente o diagnóstico bioquímico é retardado no Brasil, ocorrendo geralmente entre 24 e 96 meses $^{7}$, já no relato apresentado o atraso diagnostico foi ainda maior, ocorrendo apenas com 132 meses. A paciente também apresenta o mesmo padrão dos doentes deste artigo que passaram por vários especialistas e procedimentos cirúrgicos antes do diagnóstico ${ }^{7}$.
Um estudo apresentando no XVI Encontro de Geneticistas do Rio Grande do Sul evidenciou que dos 88 pacientes com MPS VI avaliados, a mediana de idade ao diagnóstico foi 76 meses no nordeste, 52 meses no sudeste e 32 meses no sul ${ }^{19}$. Também revelando menor atraso diagnóstico em relação à paciente deste relato.

\section{CONCLUSÃO}

Apesar da mucopolissacaridose tipo VI ser uma doença rara, suas características clínicas são precoces e geralmente evidentes. Entretanto, como demonstrado na literatura e neste relato, o diagnóstico muitas vezes é tardio, possibilitando a progressão das complicações. Por esse motivo, existe a necessidade do conhecimento da doença, para que o tratamento adequado seja iniciado precocemente, proporcionando a estes pacientes uma qualidade de vida o mais próximo possível da normalidade.

\section{REFERÊNCIAS}

1.Azevedo, ACMM. Mucopolissacaridose tipo VI: Um estudo Clínico e Radiológico Visando a Identificação de Fatores Associados à Gravidade da Doença (Tese de Doutorado). Porto Alegre: Universidade Federal do Rio Grande do Sul, 2007, 203p.

2.Mucopolissacaridoses: Manual de Orientações (endereço na internet). São Paulo: Escola Paulista de Medicina (última atualização: 08/2002; acessado em: 06/2010). Disponível em http://www.unifesp.br/centros/creim/downloads/gzmps-apostila-2003.pdf

3.Harmatz, P. Entering a new treatment age for mucopolysaccharidosis VI disease: a search for better markers of disease progression and response to treatment. J Pediatr (Rio J) 2008;84:103-6.

http://dx.doi.org/10.2223/JPED.1768

http://dx.doi.org/10.1590/S0021-75572008000200003

4.Giugliani R, Harmatz P, Wraith JE. Management Guidelines for Mucopolysaccharidosis VI. Pediatrics 2007;120;405-18.

http://dx.doi.org/10.1542/peds.2006-2184

5.Paula AC, Bertola DR, Albano LMJ, Santos ACGM, Donato FJ, Oliveira LAN, Kim CA. Achados radiológicos em pacientes com mucopolissacaridose tipo VI (síndrome de Maroteaux-Lamy). Rev imagem 2006,28:7-12.

6.Costa MI, Sabatini R, Pesquero JB, D’Almeida V, Martins AM, Soares VRX, et al. Família brasileira do nordeste apresentando co-ocorrência de MPS VI e Albinismo. 55 Congresso Brasileiro de Genética. Anais. Águas de Lindóia/SP, 2009. 7.Vieira T, Schwartz I, Muñoz V, Pinto L, Steiner C, Ribeiro M, et al. Mucopolysaccharidoses in Brazil: What Happens From Birth to Biochemical Diagnosis? Am J Med Genet 2008;146A:1741-7.

http://dx.doi.org/10.1002/ajmg.a.32320

8.Turtelli, CM. Manifestaçóes Radiológicas da Mucopolissacaridose tipo VI. Rev Radiol Bras 2002;35:311-4.

http://dx.doi.org/10.1590/S0100-39842002000500013 
9. Borges MF, Tavares FS, Silva PCL, Oliveira ZAR, Ballarin MAS, Gomes RA, et al. Mucopolissacaridose Tipo VI (Síndrome de Maroteaux-Lamy): Avaliação Endócrina de Três Casos. Arq Bras Endocrinol Metab 2003;47:87-94.

10.Canêdo MGRR, Almeida LNF, Silva RG, Almeida RNF, Alessandri EF. Pseudoglaucoma em mucopolissacaridose tipo VI: relato de caso. Arq Bras Oftalmol 2006;69:933-5. http://dx.doi.org/10.1590/S0004-27492006000600026

11.Albano LMJ, Sugayama SMM, Bertola DR, Andrade CEF, Utagawa CY, Puppi F, et al. Clinical and laboratorial study of 19 cases of mucopolysaccharidoses. Rev Hosp Clín Fac Med S Paulo 2000;55:213-8.

12.Cardoso-Santos A, Azevedo AC, Fagondes S, Burin MG, Giugliani R, Schwartz IV. Mucopolysaccharidosis type VI (Maroteaux-Lamy syndrome): assessment of joit mobility and grip and pinch strength. J Pediatr (Rio J) 2008;84:130-5.

13.Kim KH, Decker C, Burton BK. Successful Management of Difficult Infusion-Associated Reactions in a Young Patient With Mucopolysaccharidosis Type VI Receiving Recombinant Human Arylsulfatase B (Galsulfase [Naglazyme]) Pediatrics 2008;121;e714-7.

http://dx.doi.org/10.1542/peds.2007-0665

14.Herskhovitz E, Young E, Rainer J, Hall CM, Lidchi V, Chong K, et al. Bone marrow transplantation for Maroteaux-Lamy syndrome (MPS VI ): long-term follow-up. J Inherit Metab Dis 1999;22:50-62.

http://dx.doi.org/10.1023/A:1005447232027

15.Harmatz P, Whitley CB, Waber L, Pais R, Steiner R, Plecko B, et al. Enzyme replacement therapy in mucopolysaccharidosis VI (Maroteaux-Lamy syndrome). J Pediatr. 2004;144:574-80. http://dx.doi.org/10.1016/j.jpeds.2004.03.018

16.Harmatz P, Ketteridge D, Giugliani R, Guffon N, Teles EL, Miranda, MC, et al. MPS VI Study Group. Clinical Study of Recombinant Human N-Acetylgalactosamine 4-Sulfatase (Maroteaux-Lamy Syndrome): Results After 48 Weeks in a Phase 2 Open-Label During Enzyme-Replacement Therapy of Mucopolysaccharidosis VI Direct Comparison of Measures of Endurance, Mobility, and Joint Function. Pediatrics 2005;115;e681-9.

http://dx.doi.org/10.1542/peds.2004-1023

17.Harmatz P, Giugliani R, Schwartz I, Guffon N, Teles EL, Miranda, MC, et al. MPS VI Phase 3 Study Group. Enzyme replacement therapy for mucopolysaccharidosis VI: A phase 3, randomized, double-blind, placebo-controlled, multinational study of recombinant human $\mathrm{N}$-acetylgalactosamine 4-sulfatase (recombinant human arylsulfatase B or rhASB) and follow-on, open-label extension study. J Pediatr 2006;148:533-9.

http://dx.doi.org/10.1016/j.jpeds.2005.12.014

18. Harmatz P, Giugliani R, Schwartz I, Guffon N, Teles EL, Miranda MC, et al. Long-term follow-up of endurance and safety outcomes during enzyme replacement therapy for mucopolysaccharidosis VI: Final results of three clinical studies of recombinant human N-acetylgalactosamine 4-sulfatase. Mol Gen Metab 2008;94:469-75.

http://dx.doi.org/10.1016/j.ymgme.2008.04.001

19. Garcia DT, Federhen A, Himus T, Silva RF, Giugliani R, Schwartz I. Mucopolissacaridoses I, II e VI: Estudo epidemiológico comparativo entre as Regiôes Nordeste (NE), Sudeste (SE) e Sul (S) do Brasil. XVI Encontro de Geneticistas do Rio Grande do Sul. Anais. Porto Alegre, 2008. 